\title{
Identification of DNA markers associated with starch granules morphology of Solanum tuberosum L.
}

\author{
Erst T.V. ${ }^{1 *}$, Rozanova I.V. ${ }^{1}$, Khlestkin V.K. ${ }^{1,2}$, Khlestkina E.K. ${ }^{1,3}$ \\ ${ }^{1}$ Institute of Cytology and Genetics, SB RAS, Novosibirsk, Russia \\ ${ }^{2}$ All-Russia Research Institute of Farm Animal Genetics and Breeding- \\ Branch of the L.K. Ernst Federal Science Center for Animal Husbandry, St. Petersburg, Russia \\ ${ }^{3}$ N.I. Vavilov All-Russian Institute of Plant Genetic Resources (VIR), St. Petersburg, Russia \\ *e-mail: erst@bionet.nsc.ru
}

Polymers comprising raw starch granules are packed in a layered structure consisting of alternating crystalline and amorphous layers. Study of the morphological parameters of granules can provide a deeper understanding of the biochemical mechanisms of their formation and reactivity during (bio) chemical transformations. Morphology and size of starch granules are supposed to be primarily determined by starch biosynthesis genes, in particular, genes that encode SBEI and SBEII enzymes (Starch Branching Enzyme). However, it can be assumed that the set of genes affecting the morphology of the granules is wider than the structural genes of starch biosynthesis. The aim of this work is to search for genomic regions associated with parameters defining morphology of potato starch granules with genome-wide association studies (GWAS) using the Illumina 22K SNP potato array-genotyped collection (90 cultivars) from ICG "GenAgro" collection. Evaluation of starch granules was carried out on such parameters as the area, aspect ratio, circularity, roundness, feret, min. feret, solidity by the method described previously [1]. The data obtained as a result of phenotyping and genotyping were processed using Microsoft Excel, Tassel 5 and the R software. Currently, analysis for the granules circularity is completed. Parameter "circularity" characterizes how elongated the granules are. Granules with the same projection area may vary from round to elongated (from 0 to 1 ). For the studied potato starch samples parameter "circularity" varies in the range from 0.79 (cultivar Ladozhsky) to 0.87 (perspective line G.3-43-6). Data analysis with the use of GLM (Generalized Linear Model) + PCA (Principal Component Analysis) revealed a significant association with SNP located on chromosome 11. A detailed study of the identified genomic region is conducted in order to create proper diagnostic DNA-marker for further accelerated selection of plants with the required values of morphological starch granules parameters.

Acknowledgements: The study is supported by the RFBR (No. 17-29-08006).

\section{References}

1 Khlestkin V.K., Erst T.V. A practical guide to the starch granules' morphology study by microscopy. Vavilovskii Zhurnal Genetiki i Selektsii = Vavilov Journal of Genetics and Breeding. 2017;21(6):728-734. DOI 10.18699/VJ17.290 (in Russian). 